IPSI Revista de Investigación en Psicología 1998, Vol. 1, № 2, pp. 113 - 133

\title{
ESTRÉS Y AFRONTAMIENTO DEL ESTRÉS FRENTE AL EXAMEN EN EDUCACIÓN SUPERIOR: ¿UN ESTILO O UN PROCESO?
}

\author{
Alberto Quintana Peña
}

Se han contrastado dos enfoques teórico-metodológicos a fin de identificar los factores que explicarían las variables dependientes: a) estrés experimentado frente a los exámenes, y b) modos de afrontamiento de tal estrés. Se argumenta que, mientras los teóricos del Yo asumen como variable independiente de la ansiedad frente a los exámenes la Ansiedad-rasgo, y como variables independientes de los modos de afrontamiento el estrés, el estilo de orientación personal (locus de control interno-externo) y el estilo cognitivo (dependenciaindependencia de campo); de otro lado, los estudiosos de la conducta como proceso consideran como variables independientes de la primera a la evaluación cognitiva del examen y de los segundos a las etapas del proceso de estrés (previa y después del examen). La muestra comprendió 236 adolescentes de ambos sexos, estudiantes en una academia preuniversitaria auspiciada por una universidad para el ingreso directo, con edades entre 16 y 18 años, seleccionados mediante un muestreo no probabilistico, intencional. Los resultados obtenidos permiten concluir que la explicación proporcionada por los teóricos de la conducta como proceso se corresponde más con los hechos, que la brindada por los teóricos del Yo.

Palabras Clave: Estrés, examen, alumnos preuniversitarios, modos de afrontamiento del estrés, evaluación cognitiva, etapas del proceso de estrés.

We try to compare two theoretical-methodological approaches in order to identify factors that explain the dependent variables: evaluation stress and 
coping styles to this stress. On one side, Self theorists assume as independent variable of the evaluation stress the trait anxiety toward evaluation, and as independent variables of the coping stress styles the personal orientation (locus of control internal-external), and the cognitive style (dependence-independence of field). On the other side, theorists of behavior as process consider as independent variables of the first the cognitive evaluation of the exam and for the seconds tire stages of stress process (pre and post exam stages), The sample was 236 adolescents, of both genders, students of a pre-university academy, held by an university for direct acceptance, aged 16 to 18 years, selected by non probabilistic intentional sampling. Obtained results lead to the conclusion that the explanation about stress toward exams and the coping styles given for the theorists of behavior as process has more relationship with the facts than the given by self theorists.

Key words: Stress, exams, pre-universitary, coping stress styles, cognitive evaluation, stages of stress procesess

En las sociedades contemporáneas, la competencia por el éxito es posiblemente la fuente principal de estrés (May, 1950, citado por Bavermeister, 1989). Frecuentemente las oportunidades de éxito están condicionadas por la ejecución de las personas en pruebas o exámenes.

Para ser aceptado en la universidad, un estudiante necesita tener muy buenas calificaciones, además de obtener buenos resultados en difíciles exámenes de admisión. Por ello no es sorprendente que los exámenes sean evaluados como una amenaza, generando reacciones emocionales de ansiedad y malestar. De aquí se explica que la ansiedad producida por las pruebas sea un problema común entre los estudiantes de secundaria y postulantes a la universidad.

La mayoría de los estudiantes perciben el momento del examen como algo amenazador y experimentan un incremento de su estado de ansiedad frente al mismo. En general, cualquier examen difícil somete a los candidatos a dos situaciones: la dificultad intrínseca de las preguntas y el posible nivel distorsionador de la ansiedad. Se ha comprobado que mientras un incremento moderado en el nivel de ansiedad puede ser útil si motiva al estudiante a incrementar sus esfuerzos y a enfocar su atención en el contenido del examen, un mayor incremento de la ansiedad puede producir resultados insatisfactorios. Los efectos debilitantes de estas 
reacciones emocionales sobre la ejecución en los exámenes han sido ya demostrados (García, 1983).

Frente a estos hechos encontramos que las diversas teorías y aproximaciones metodológicas pueden ser agrupadas en dos enfoques diametralmente opuestos:

De un lado, los "teóricos del Yo", para quienes el concepto de ansiedad frente al examen ha sido definido como un rasgo de personalidad específico frente a esa situación (Spielberger, 1980). Las investigaciones (Sarason, 1960, 1972, 1975; Wine, 1971; Alpert y Haber, 1960; Mandlery y Sarason, 1952; citados por Bavermeister, 1989) sugieren que este concepto se refiere a diferencias individuales en la propensión a tener reacciones de ansiedad frente a los exámenes. Estos estudiosos sostienen que al enfrentarse a una situación de evaluación, las personas con el rasgo de ansiedad frente a los exámenes son más propensas a tener reacciones de ansiedad-estado y cogniciones de preocupación egocéntricas (Morris y Liebert, 1970; Sarason, 1960, 1972, 1975; Spielberger, Anton y Bedell, 1976, citados en Spielberger, 1980). Las reacciones de ansiedad-estado se caracterizan por sentimientos conscientemente percibidos de aprehensión (temor) y nerviosismo, y por la activación del sistema nervioso autónomo. Las cogniciones de preocupación egocéntrica se refieren a pensamientos autoevaluativos no pertinentes a la tarea, de tipo autocrítico y autodestructivo (Morris y Liebert, 1970; Sarason, 1960, 1972, 1975; Spielberger y cols., 1976; Wín , 1971, citados en Bavermeister, 1989).

De otro lado, se encuentran los teóricos de la conducta como proceso (fig. 1), para quienes los acontecimientos de la vida no provocan problemas serios en la mayoría de las personas cuando ocurren a su debido tiempo y son esperados. Lo estresante en ellos es la importancia que el sujeto otorgue al cambio o a su ausencia, lo cual dependerá de la propia historia personal, de la etapa de la vida en la que se encuentre y del conjunto de circunstancias presentes (Lennon, 1982, Stewart, 1982, citados por Lazarus y Folkman, 1986). En este contexto, Lazarus (1966) define el estrés como la emoción, la motivación o la cognición que resulta de la evaluación que el sujeto hace de aquellas relaciones con el medio que sobrepasan sus recursos o los del sistema social; y, para el caso específico de los exámenes, esta 
evaluación estaría referida al grado de dificultad que los estudiantes esperan, la importancia que el examen tenga para cada uno, hasta qué punto piensan que podrán controlar la situación, y la posibilidad que creen tener de cambiar la relación problemática entre ellos y su entorno (Folkman y Lazarus, 1986).

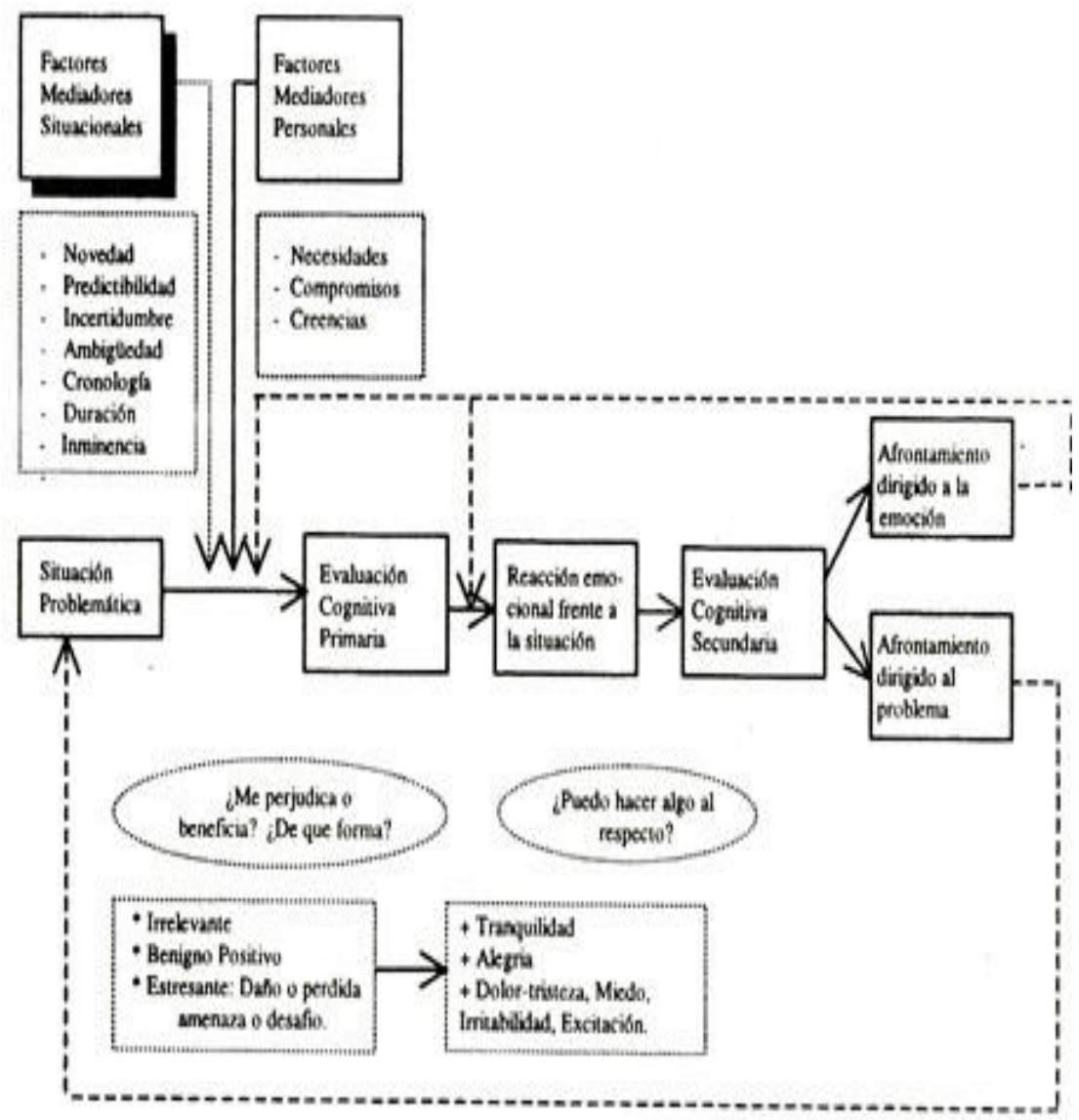


Fig. 1. Modelo del estrés como E - R (Lazarus 1996).

En el modelo teórico de la Psicología del Yo se define al afrontamiento como el conjunto de pensamientos $y$ actos realistas $y$ flexibles que solucionan problemas y, por tanto, reducen el estrés.

Diferencian entre varios procesos usados por el sujeto para manipular la relación individuo-entorno, pero se han limitado a clasificar a los sujetos para luego predecir la forma como afrontarían algunos o todos los acontecimientos estresantes con los que pudieran enfrentarse.

En este contexto se define al afrontamiento como un estilo o un rasgo, más que como un proceso dinámico del yo, (Loeving, 1976; Shapiro, 1965; Vaillant, 1977; citado en Nawas, 1971). Así, se habla de estilo de afrontamiento como una forma amplia, generalizada y abarcad()ra de referirse a tipos particulares de individuo, tales como poderoso-sin poder, amigable-hostil, controlador-permisivo, o a tipos particulares de situación, tales como ambigua-clara, inminente-alejada, temporal-crónica, evaluativano evaluativa.

En este modelo teórico se originó la teoría e investigación acerca de los estilos cognitivos, entendidos éstos como respuestas automáticas que sirven de mecanismo de control, a saber: dependencia-independencia de campo, nivelación-aguzamiento, complejidad-simplicidad, etc.; así como a la referida a los estilos de orientación personal: locus de control internoexterno, directividad interior-otros, introversión-extraversión, etc. Todos ellos derivados del concepto central en la psicología de Adler: el estilo de vida (Nawas, 1971).

En cambio para los teóricos de la conducta como proceso (fig. 1), la calidad unidimensional de la mayoría de conceptualizaciones y mediciones de rasgos y estilos no reflejan adecuadamente la complejidad y variabilidad de la naturaleza multidimensional de los procesos de afrontamiento utilizados para enfrentarse a las situaciones de la vida real (Mechanic, 1962; Murphy, 1974; Vigotsky y cols. 1961; citados por Lazarus y Folkman, 1986). Y agregan que el afrontamiento es aquel subconjunto de actividades adaptativas que implican esfuerzo, y no todo aquello que 
hacemos en relación al entorno. Así, los estilos cognitivos pueden considerarse procesos adaptativos, pero no de afrontamiento. De esta forma definen el afrontamiento como aquellos esfuerzos cognitivos y conductuales constantemente cambiantes que se desarrollan para manejar las demandas específicas externas y/o internas que son evaluadas como que están excediendo o desbordando los recursos del individuo.

\section{FORMULACIÓN DEL PROBLEMA}

De las consideraciones señaladas se desprende que la finalidad del presente trabajo de investigación es examinar la validez de los conceptos y teorías elaboradas por los investigadores en el enfoque de los teóricos del Yo en contraste a los elaborados por los investigadores en el enfoque de la conducta como proceso, tanto respecto al estrés y al afrontamiento del estrés en general, como en cuanto al estrés y su afrontamiento ante la situación de examen en una población de postulantes a estudios de educación superior. Para ello se plantean las siguientes interrogantes:

¿El grado de estrés experimentado por los sujetos frente al examen estará en función del nivel en que presentan el rasgo - "ansiedad frente a los exámenes" o, por el contrario, de la "evaluación cognitiva" que se haga del examen como - más o menos estresante?

¿La elección de los modos de afrontamiento del estrés que emplean los sujetos frente a los exámenes dependerá de su "estilo de afrontamiento" (cognitivo, de orientación interpersonal), o, por el contrario, de la "etapa del proceso de estrés" en que se encuentran (antes o después del examen)?

\section{OBJETIVOS}

Conocer cuál de los dos enfoques teórico-metodológicos examinados. El del Yo o el de la conducta, explica de manera más exhaustiva los hallazgos respecto al estrés frente a los exámenes y los modos en que éste es afrontado.

Describir los niveles de ansiedad y los modos de afrontamiento del estrés que presentan las personas frente al examen de admisión a la 
educación superior.

Aportar datos para la elaboración de programas de entrenamiento en modos de afrontamiento del estrés, aplicables a los alumnos de los últimos años de secundaria a través de los servicios de Orientación y Bienestar del Educando.

\section{HIPÓTESIS}

No existe una relación significativa entre él estrés experimentado frente al examen (ansiedad-estado), y la ansiedad de rasgo frente a los exámenes (ansiedad-rasgo).

Existe una relación significativa entre el estrés experimentado frente al examen y la evaluación del examen como estresante.

No existe diferencia significativa en los modos en que afronta el estrés el grupo de sujetos con estilo cognitivo "dependiente de campo" en comparación al grupo con estilo cognitivo "independiente de campo".

No existe diferencia significativa en los modos en que afronta el estrés el grupo de sujetos con estilo de orientación personal "locus de control interno" en comparación al grupo con estilo de orientación personal "locus de control externo".

Existe diferencia significativa en los modos en que los sujetos afrontan el estrés en la etapa previa al examen en comparación a los modos en que lo hacen después del examen y antes de recibir la calificación correspondiente.

\section{IMPORTANCIA DEL ESTUDIO}

En los últimos años es preocupante la cada vez más frecuente presencia de conceptos referidos a los rasgos y estilos en las investigaciones de los psicólogos peruanos (Meza, 1987, 1988; Lazarte, 1982), soslayando el hecho que con estos estudios no hacen otra cosa que dividir una población heterogénea en subgrupos homogéneos que sólo permiten la relación entre un predictor y un criterio. Estos intentos, al simplificar de una forma 
grosera patrones complejos reduciéndolos a esquemas unidimensionales tendrían poco valor explicativo y predictivo de lo que hace realmente el sujeto en cada contexto.

Frente a esto, resulta conveniente estudiar la propuesta de Folkrnan y Lazarus (1986), quienes intentan estudiar el afrontamiento recurriendo a la evaluación directa de las conductas de afrontamiento y a la forma en que cambian a medida que lo hacen las demandas de la situación y las evaluaciones que el individuo hace de estas.

\section{METODOLOGÍA}

\section{Método de investigación}

El presente trabajo de investigación es de carácter ex-post-facto (Craig y Metze, 1982), supone la implementación repetida de varios diseños univariables bivalentes. (Castro, 1977), a continuación se presenta un cuadro que resume la relación entre las variables investigadas de acuerdo al problema planteado, en 61 se explican las variables independientes no manipuladas $(\mathrm{X})$ que explican las variables dependientes $\mathrm{O}$, según los enfoques contrastados (cuadro 1).

\section{Población}

La población objetivo está integrada por jóvenes peruanos, de ambos sexos, postulantes a estudios de educación superior, en situación de examen de admisión.

\section{Muestra}

\section{Selección de la muestra}

La técnica utilizada para seleccionar la muestra fue el muestreo no probabilístico intencional (Alarcón, 1991, Kerlinger, 1991), debido a que no se ha conocido la posibilidad de cada uno de los elementos de la población de poder ser seleccionados en la muestra, y han sido elegidos en función de cumplir con los criterios de inclusión o características de la 
muestra.

\section{Cuadro 1. Variables investigadas según los enfoques teórico- metodológicos contrastados}

\begin{tabular}{|c|c|c|}
\hline \multirow{2}{*}{$\begin{array}{c}\text { Variables } \\
\text { Dependientes }\end{array}$} & \multicolumn{2}{|c|}{ Variables Independientes por Enfoque } \\
\cline { 2 - 3 } & Teóricos del Yo & $\begin{array}{c}\text { Teóricos de la } \\
\text { Conducta }\end{array}$ \\
\hline Ansiedad-estado & Ansiedad-rasgo & $\begin{array}{c}\text { Evolución } \\
\text { Cognitiva }\end{array}$ \\
O & $(X)$ & $(X)$ \\
\hline Modos de & Estilos Cognitivos: & $\begin{array}{c}\text { Etapa del Proceso } \\
\text { de examen: }\end{array}$ \\
afrontamiento: & Dependiente- & $-\quad$ Etapa previa \\
A la emoción & independiente & al examen \\
Al problema & de campo & Después del \\
& $(X)$ & examen \\
Control Interno- & $(X)$ \\
& Externo & \\
& $(X)$ & \\
\end{tabular}

\section{Características de la muestra}

La muestra estuvo conformada por 236 jóvenes de 16 a 18 años de edad, de ambos sexos (que luego de la depuración se redujeron a 97,46 varones y 51 mujeres), residentes de Lima, estudiantes de una academia preuniversitaria. Jóvenes que se encontraban rindiendo los exámenes de selección, exámenes de cuyos resultados dependía su ingreso a la universidad promotora de la academia (por ejemplo: "pre-San Marcos", "pre-Agraria", "pre-Cayetano", "pre-Católica, etc).

\section{Variables de investigación}

En tanto se busca confrontar dos enfoques teórico-metodológicos 
respecto a las variables que explicarían la presencia de ansiedad frente a los exámenes y los modos de afrontarla que serán elegidos, (ver esquemas 2 y 3), agrupemos las variables de investigación 
Según estos dos problemas

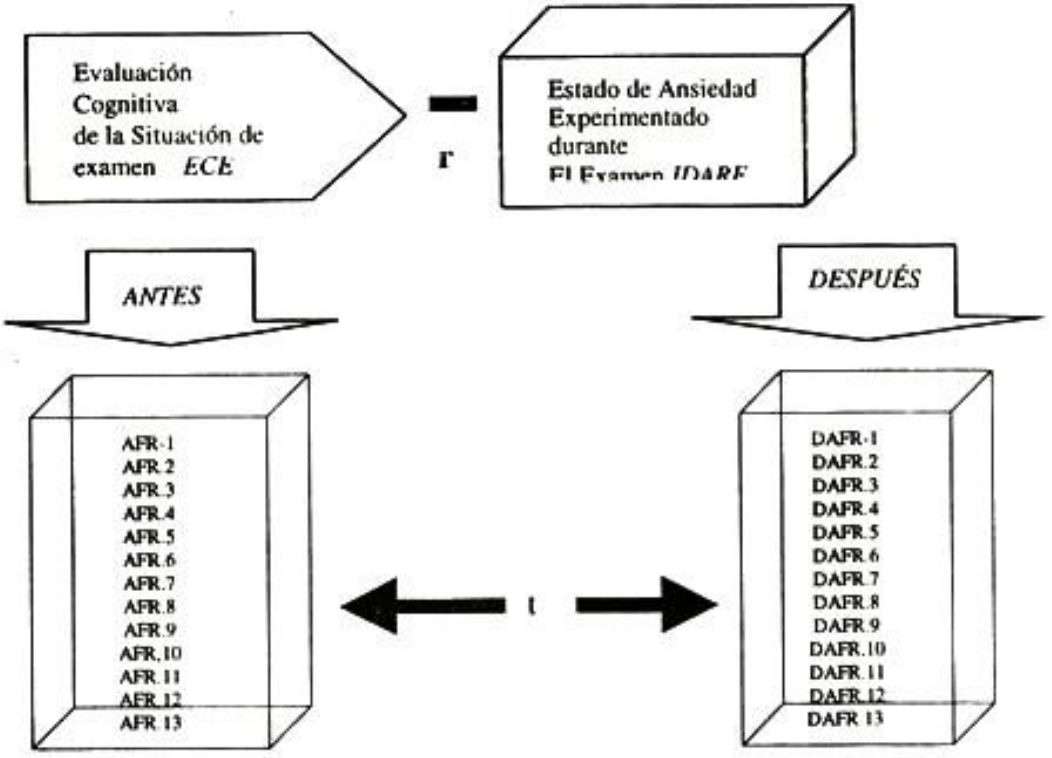

Figura 2. Modelo de la relación entre las variables postulado por los téricos de la conducta como proceso.

\section{Definición de variables}

\section{a) Para el estudio del estrés experimentado frente al examen}

Variable Dependiente: Estrés frente al examen: Estado emocional transitorio, consistente en percibir el momento del examen como algo amenazador y experimentar un incremento del estado de ansiedad durante su ejecución. Es medida por el grado de acuerdo de los sujetos con los 20 reactivos referidos a ansiedad-estado del Inventario de Autoevaluación de 
la ansiedad Rasgo-Estado (IDARE) administrada la noche del día anterior al examen. Estos son reactivos tipo Likert, politómicos, de calificación 1-4/4-1, evaluadores de la ansiedad experimentada por los sujetos en ese mismo momento (Spielberger, y Col, 1975).

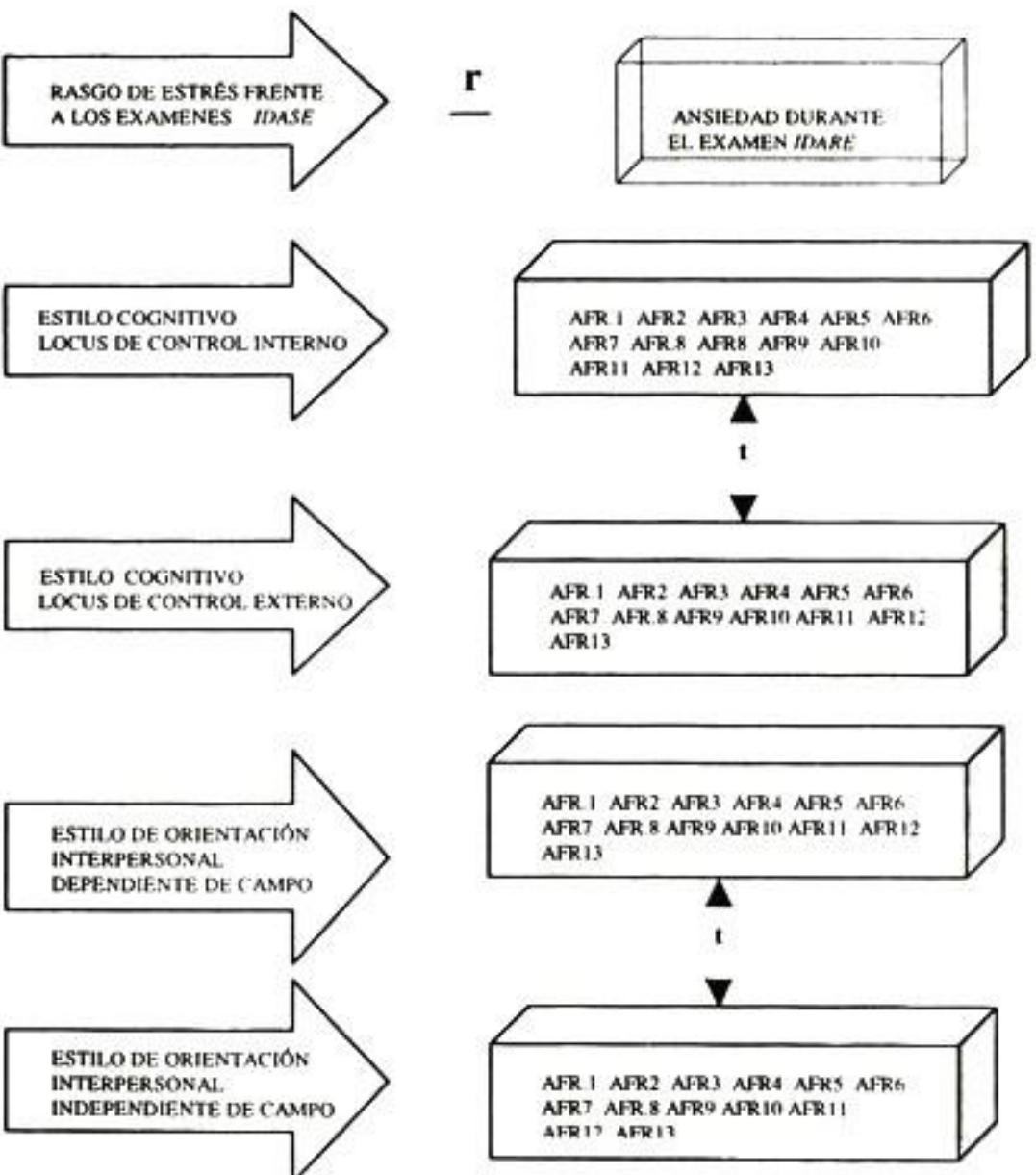

Figura 3. Modelo de la relación entre las varables postulado por los té́ricos del Yo. 
Variable Independiente ${ }_{1}$ : Rasgo de ansiedad frente a los exámenes: Propensión a tener reacciones de ansiedad-estado y cogniciones de preocupación egocéntricas. Las reacciones de ansiedad- estado se caracterizan por sentimientos conscientemente percibidos de aprehensión (temor) y nerviosismo y por la activación del sistema nervioso autónomo. Las cogniciones de preocupación se refieren a pensamientos autoevaluativos no pertinentes a la tarea, de tipo autocrítico y autodestructivo. Medida por el grado de acuerdo de los sujetos con los ocho reactivos tipo Likert, politómicos de calificación 1-4/4-1, evaluadores de la ansiedad ante el examen del Inventario de Autoevaluación sobre Exámenes (IDASE) (Bavermeister, Collazo y Sielberger, 1983, citado en Bavermeister, 1988).

Variable Independiente ${ }_{2}$ : Evaluar el examen como estresante: Proceso que determina las repercusiones y consecuencias que el examen provocará en el individuo, por ejemplo: ¿Me perjudica o beneficia, ahora o en el futuro, y de qué forma?, ¿Puede hacerse algo al respecto? Estimada por el grado de acuerdo de los sujetos con siete reactivos de formato tetrádico y de calificación 1-0, desarrollada ad-hoc para facilitar un autoinforme estandarizado de la evaluación. que se realiza de las repercusiones y consecuencias que cada estudiante espera del examen (Quintana, 1998). Todas serían variables ordinales.

\section{b) Para el estudio de los modos de afrontamiento al estrés}

Variable Dependiente: Modos de afrontamiento al estrés: Aquellas estrategias destinadas a modificar una situación evaluada como de daño, pérdida, amenaza o desafío, o en aquellas situaciones estresantes que no pueden modificarse, aquellas secuencias de decisiones (estrategias) que permiten al sujeto tolerar, minimizar, aceptar o incluso ignorar aquello que no puede modificar, (Variable Dependiente). Medida por el grado de acuerdo de los sujetos con 52 reactivos tipo Lickert, poltómicos, de 
calificación 1-4, evaluadores de las acciones observables o encubiertas con las cuales se afronta la situación de estrés experimentada en ese mismo momento (Carver y cols., citado por Salazar, 1993).

\section{Variable Independiente ${ }_{1}$ :}

Locus de control interno-externo: Dimensión de la personalidad caracterizada porque las personas "interiores" creen que su comportamiento es lo que controla sus refuerzos, mientras que las personas "exteriores" creen que es algo más lo que determina si su comportamiento ha de ser reforzado o no (por ejemplo: el destino, la suerte, la casualidad). Estimada por el grado de acuerdo de los sujetos con los 15 reactivos de elección forzada y calificación dicotómica 1-0, evaluadores del locus (lugar) de control de la Escala I-E (Rotter, 1966).

\section{Variable Independiente ${ }_{2}$ :}

Dependencia-independencia de campo: Una forma analítica de percepción en contraste con una forma global, que supone una forma particular de comprender los estímulos generados por el entorno, y que en el caso de la independencia de campo, refleja la capacidad de sobreponerse a la influencia de un contexto sólido; es decir, estructurado (variable Independiente). Estimada por el éxito o fracaso en descubrir y delinear con un lápiz una figura simple al interior de una figura compleja en los 18 reactivos de calificación dicotómica 1-0, evaluadores de la independenciadependencia de campo, de la "prueba de figuras anidadas" (Witkin y Col., citado por Meza, 1987).

\section{Variable Independiente : $_{3}$}

Proceso del estrés frente al examen etapa previa - etapa posterior:

Se refiere a las múltiples demandas existentes en un examen en tanto situación estresante y a los cambios que éstas van sufriendo a medida que transcurre el tiempo, (Variable Independiente). Estimada observando a los sujetos en la etapa previa y en la etapa posterior al examen estresante. 
Etapa previa o de preparación: dos días antes del examen. Etapa posterior O de adecuación a los resultados: cinco días después del examen y antes de recibir los resultados.

Todas serían variables ordinales.

\section{PROCEDIMIENTO}

En primer lugar se hicieron las coordinaciones necesarias con quienes se responsabilizan de las acciones de orientación del estudiante en la Academia Pre-universitaria, a fin de administrar los instrumentos como parte de sus acciones previas a la orientación. Lograda la aceptación se administraron a los sujetos integrantes de diez aulas (236 alumnos de ambos sexos), que cumplían los criterios especificados para la muestra, la Escala Abrevia4a De Control Interno-Externo, la Prueba De Figuras Anidadas y la Escala De Autoevaluación De La Ansiedad Producida Por Los Exámenes (IDASE). Tres días antes de un examen estresante se les administró el Cuestionario sobre Modos de Afrontamiento del Estrés y el Cuestionario de Evaluación Cognitiva del Examen. Un día antes del examen se les administro la escala de ansiedad-estado del IDARE. Por último, después del examen y antes de que recibieran las calificaciones correspondientes se les volvió a administrar el cuestionario sobre modos de afrontamiento del estrés. Finalizando la administración de los instrumentos se elaboro la base de datos en la hoja de calculo Microsoft Excel, depurando a los sujetos que no habían asistido a la administración de uno u otro de los instrumentos quedando la base de datos reducida a 97 sujetos, con los cuales se hizo el análisis estadístico correspondiente usando el Statistical PaekaRefor the Social Sciences (SPSS), for Microsoft Windows 6.1 .

\section{ANALISIS DE RESULTADOS}

Con el objetivo de dar respuesta a la interrogante formulada en el primer problema de investigación y contrastar las hipótesis correspondientes se efectúo una matriz de correlaciones de todas las variables en estudio, a 
excepción de los modos de afrontamiento al examen estresante (tabla 1), la misma que permitió corroborar su grado de asociación.

Hubo una significativa correlación positiva ( $r=.85$ a un nivel de significación $\mathrm{p}=.001$ ), entre la evaluación cognitiva del compromiso personal implicado en el examen estresante y la ansiedad experimentada frente al mismo (tabla 1). Es decir, a mayor evaluación cognitiva frente al examen como estresante, mayor es la ansiedad que se experimenta frente al mismo.

Tabla 1. Coeficientes de correlación de las variables en estudio a excepción de los modos de afronte del examen estresante

\begin{tabular}{|c|c|c|c|c|c|}
\hline & $\begin{array}{l}\text { Rasgo de } \\
\text { Ansiedad }\end{array}$ & $\begin{array}{c}\text { Evaluació } \\
\mathrm{n} \\
\text { Cognitiva } \\
\text { del } \\
\text { examen }\end{array}$ & $\begin{array}{c}\text { Ansiedad } \\
\text { experimentad } \\
\text { a en } \\
\text { el examen }\end{array}$ & $\begin{array}{c}\text { Locus de } \\
\text { Control } \\
\text { lnter/extem } \\
\text { o }\end{array}$ & $\begin{array}{c}\text { Estilo } \\
\text { Cognitivo } \\
\text { lndep.ldepend. } \\
\text { de } \\
\text { campo }\end{array}$ \\
\hline Rasgo de ansiedad & 1.00 & -.10 & .01 & -.12 & $-.26 * *$ \\
\hline $\begin{array}{l}\text { Evaluación Cognit. } \\
\text { Del examen }\end{array}$ & & 1.00 & $.84 * *$ & .03 & .04 \\
\hline $\begin{array}{l}\text { Ansiedad } \\
\text { experimentada en el } \\
\text { examen }\end{array}$ & & & 1.00 & .02 & .06 \\
\hline $\begin{array}{l}\text { Locus de Control } \\
\text { Inter/extemo }\end{array}$ & & & & 1.00 & .05 \\
\hline $\begin{array}{l}\text { Estilo Cognitvo } \\
\text { Indep/depend. de } \\
\text { Campo }\end{array}$ & & & & & 1.00 \\
\hline
\end{tabular}

Hubo una significativa correlación negativa $(r=-.26)$, entre el estilo cognitivo dependiente-independiente de campo y el rasgo de ansiedad frente a los exámenes, 10 cual estaría indicando que cuanto más independiente de campo era la persona menor era el nivel del rasgo de ansiedad frente a los exámenes, o 10 que es 10 mismo, que los sujetos más dependientes de campo tendieron a presentar mayor rasgo de 
ansiedad frente a los exámenes.

Se encontró una correlación positiva nula $(r=.01)$, entre el rasgo de estrés frente a los exámenes y el estado de ansiedad experimentado durante en el mismo, (tabla 1). Lo cual indicaría que la medida del rasgo de ansiedad frente a los exámenes no daría una adecuada explicación de la ansiedad esperada en una situación de examen específica.

Resulta llamativo observar que mientras ni el rasgo de ansiedad frente a los exámenes, ni el estilo cognitivo independencia-dependencia de campo, ni tampoco el locus de control interno-externo, mantienen una correlación significativa con los modos de afrontamiento al estrés en la etapa previa al examen, por el contrario, la evaluación cognitiva de éste como estresante y la ansiedad experimentada frente al mismo presentan correlaciones significativas con estos modos, en su mayoría superiores a $r=.70$, (tabla 2$)$.

Tabla 2. Coeficientes de las correlaciones entre lo modos de afronte usados en la etapa previa al examen estresante y todas las variables usadas en la investigación ( $n=97)$.

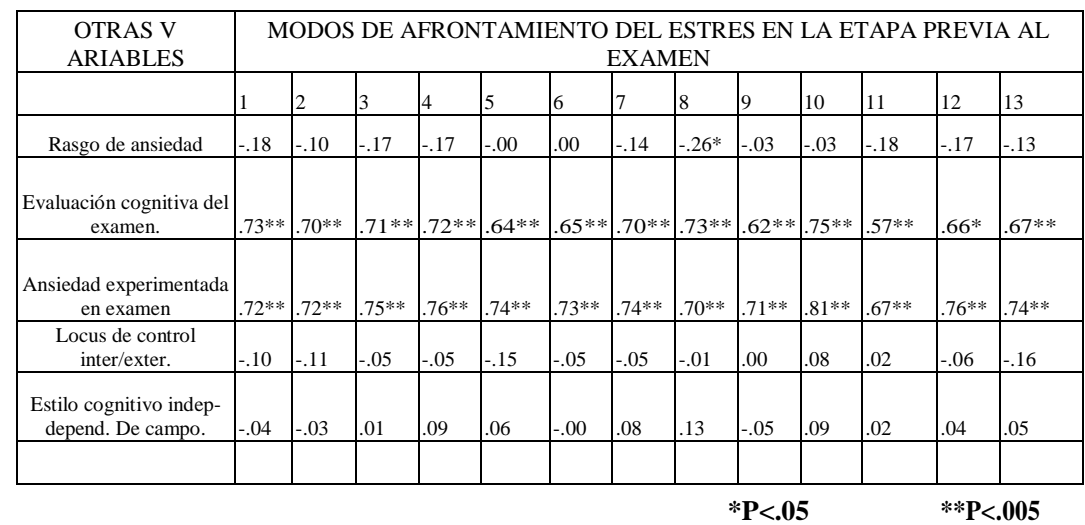

Diferencia de medias para muestras independientes definiendo los grupos en función al hecho de haber obtenido una puntuación alta (puntaje $<=$ al percentil 75) o baja (puntaje $<=$ al percentil 25), en una variable de referencia. 
Al comparar al grupo de sujetos dependientes de campo (puntajes bajos en la prueba de figuras ocultas) con el grupo de los independientes de campo (puntajes altos), en cuanto a cada uno de los modos de afrontamiento al estrés, no se encontró ni una sola diferencia significativa entre ellos, (Quintana, 1998).

Así también al comparar al grupo de sujetos con locus de control externo (puntajes altos en la prueba de locus de control) con el grupo de sujetos con atribuciones de control interno (puntajes bajos), en cuanto a cada uno de los modos de afrontamiento al estrés, tampoco se encontró ni una sola diferencia significativa entre ellos, (Quintana, 1998).

Diferencia de medias para muestras relacionadas comparando el uso promedio que hicieron los sujetos de un determinado modo de afrontamiento tanto en la etapa previa como después del examen estresante.

Se encontró, coincidiendo con lo afirmado por los teóricos de la conducta, que hubo diferencias significativas en el uso de todos los modos de afronte al estrés que se hizo en la etapa previa al examen comparado con el que se hizo después, (tabla 3).

\section{DISCUSIÓN}

En relación a las variables que explican la ansiedad experimentada por los sujetos frente a los exámenes estresantes, los datos llevan a rechazar la primera hipótesis y a afirmar: que no existe una relación significativa entre el estrés experimentado frente al examen (ansiedad-estado), y la ansiedad de rasgo frente a los exámenes, y a la vez a aceptar la segunda hipótesis, es decir que existe una relación significativa entre el estrés experimentado frente al examen y la evaluación del examen como estresante. Lo que es consistente a los postulados de Lazarus (1986), en el sentido de que el estrés sería la ansiedad resultante de la evaluación que los sujetos han hecho de sus recursos para interactuar con el entorno (recursos que perciben como insuficientes), del grado de dificultad que esperaban en el examen (difícil), de la importancia que éste tenía para cada uno (muy importante), que tanto esperaban controlar la situación (incontrolable), y la 
posibilidad de modificar esta reacción (imposible).

Las correlaciones significativas encontradas entre las variables modos de afrontamiento al estrés en la etapa previa al examen, evaluación cognitiva de éste como estresante y la ansiedad experimentada frente al mismo, coincide con los hallazgos de Folkman y Lazarus ( J 980, citado por Lazarus y Folkman 1986) y Krantz (J 983), Y estarían indicando que las tres variables, a nuestro juicio de estado y no de rasgo, estarían altamente asociadas.

\section{Tabla 3. Diferencia de medias ' $t$ ' comparando uno por uno los modos de afronte al estrés en cuanto a su uso en la etapas previa al examen y después del examen pero antes de recibir sus calificaciones $(\mathbf{n}=97)$}

\begin{tabular}{|c|c|c|c|c|c|c|}
\hline & \multirow{3}{*}{ Modo de afrontamiento } & \multicolumn{4}{|c|}{$\begin{array}{c}\text { Proceso de estrés frente al } \\
\text { examen }\end{array}$} & \multirow{3}{*}{$\mathrm{t}$} \\
\hline & & \multicolumn{2}{|c|}{$\begin{array}{c}\text { En la etapa } \\
\text { previa al }\end{array}$} & \multicolumn{2}{|c|}{$\begin{array}{c}\text { Después del } \\
\text { examen }\end{array}$} & \\
\hline & & Media & DS & Media & DS & \\
\hline 1 & Afronte Activo & 9.84 & 4. & 11.10 & 1.65 & $-2.97 * *$ \\
\hline 2 & Planificación & 9.99 & 4. & 11.92 & 2.10 & $-4.43 * *$ \\
\hline 3 & Supresión de otras Actividades & 9.76 & 4. & 11.31 & 2.06 & $-3.47 * *$ \\
\hline 4 & Postergación del Afrontamiento & 9.15 & 3. & 10.66 & 1.50 & $-3.79 * *$ \\
\hline 5 & $\begin{array}{l}\text { Búsqueda de apoyo social por razones } \\
\text { Instrumentales }\end{array}$ & 9.55 & $43^{4 .}$ & 10.64 & 2.77 & $-2.49 *$ \\
\hline 6 & $\begin{array}{c}\text { Búsqueda de apoyo social por razones } \\
\text { emocionales }\end{array}$ & 9.45 & $40^{4 .}$ & 10.48 & 2.98 & $-2.19 *$ \\
\hline 7 & $\begin{array}{l}\text { Reinterpretación positiva y crecimiento } \\
\text { personal }\end{array}$ & 11.06 & $56^{4 .}$ & 12.79 & 1.72 & $-3.54 * *$ \\
\hline 8 & Aceptación & 9.38 & 4. & 11.27 & 2.44 & $-4.12 * *$ \\
\hline 9 & Acudir a la Religión & 9.25 & 4. & 10.31 & 3.17 & $-2.45 *$ \\
\hline 10 & Focalizarse en la emoción y liberarla & 8.77 & 3. & 10.03 & 2.11 & $-3.03 * *$ \\
\hline 11 & Negación & 7.12 & 3. & 8.24 & 2.30 & $-3.01 * *$ \\
\hline 12 & Renuncia conductual al afrontamiento & 6.19 & 3. & 6.95 & 2.15 & $-2.47 *$ \\
\hline 13 & Renuncia mental al afrontamiento & 8.29 & $77^{3 .}$ & 9.60 & 2.24 & $-3.27 * *$ \\
\hline
\end{tabular}


En relación a las variables que explican los modos en que los sujetos afrontan el estrés frente a los exámenes, los resultados son concluyentes en cuanto a rechazar la tercera hipótesis y a afirmar que no existen diferencias significativas en los modos de afrontamiento de los grupos de sujetos en función a sus estilos cognitivos (independencia-dependencia de campo). Lo que contradice las afirmaciones de los teóricos del Yo a este respecto pero coincide con las afirmaciones de teóricos de la conducta como proceso (Mechanic 1962, Murphy 1974, Vigotsky y col. 1961, citados. por Lazarus y Folkman, 1986), en el sentido de que las conceptualizaciones y mediciones de estilos no reflejan adecuadamente la complejidad y variabilidad de los procesos de afrontamiento utilizados para enfrentarse a las situaciones de la vida real.

Así también, los resultados son concluyentes en cuanto a rechazar la cuarta hipótesis y a afirmar que no existen diferencias significativas en los modos de afrontamiento de los grupos de sujetos en función a sus estilos de orientación personal (locus de control interno/externo). Lo que una vez más contradice las afirmaciones de los teóricos del Yo al respecto y coincide con los resultados de las investigaciones de los teóricos de la conducta como proceso, Folkman, Alduiwn y Lazarus (1981, citados en Lazarus y Folkman, 1986), quienes en una población de adultos (45 a 65 años), encontraron que las creencias generales acerca del locus de control no guardan relación con los modos de afrontamiento.

En cuanto a nuestra cuarta hipótesis los resultados son concluyentes respecto a aceptar la hipótesis; es decir, que existen diferencias significativas en los modos de afrontamiento al estrés usados por los sujetos en la etapa previa del examen en comparación con los usados en la etapa posterior. 


\section{CONCLUSIONES}

1. No existe una relación significativa entre el experimentado frente al examen (ansiedad-estado), ansiedad de rasgo frente a los exámenes.

2. No existen diferencias significativas en los modos de afrontamiento de los grupos de sujetos en función a sus estilos cognitivos (independenciadependencia de campo).

3. No existen diferencias significativas en los modos de afrontamiento de los grupos de sujetos en función a sus estilos de orientación personal (locus de control interno/externo).

4. Existe una relación significativa entre el estrés experimentado frente al examen y la evaluación del examen como estresante.

5. Existen diferencias significativas en los modos de afrontamiento al estrés usados por los sujetos en la etapa previa del examen en comparación con los usados en la etapa posterior es decir los sujetos tienen la tendencia en promedio a no usar los modos de afrontamiento en la misma proporción en las etapas antes y después del examen.

6. Las variables modos de afrontamiento al estrés, evaluación cognitiva de éste como estresante y la ansiedad experimentada frente al mismo muestran una alta correlación lo cual estaría indicando que las tres variables, a nuestro juicio de estado, estarían altamente asociadas.

7. En pocas palabras podemos decir que la explicación de los teóricos de la conducta como proceso de la ansiedad experimentada durante un examen estresante y los modos de afronte desplegados frente a ella, dan perfecta cuenta de los hallazgos encontrados en una muestra de jóvenes peruanos preuniversitarios, mientras que una explicación basada en las teorías de los rasgos y estilos fracasa en hacerla. 


\section{BIBLIOGRAFÍA}

Alarcón, R. (1991). Métodos y diseños de investigación del comportamiento. Lima. UPCH.

Bavermeister, J. (1986). Confiabilidad y validez del inventario de ansiedad rasgo y estado para niños portorriqueños y panameños. Revista interamericana de Psicología. XX( 1-2).

Bavermeister, J. (1988). El inventario de autoevaluación sobre exámenes (IDASE) y su aplicabilidad a estudiantes de escuelas secundarias. Hispanic Journal of Behavioral Sciellces. Universidad de Puerto Rico, X,(1).

Bavermeister, J. (1989). Estrés de Evaluación y reacciones de ansiedad ante la situación de examen. Avances en Psicología Clínica Latinoamericana. V( 1), 69-88.

Castro, L. (1977). Diseño experimental sin estadística. México: Trillas.

Craig, J. y Metze, L. (1982). Métodos de la investigación psicológica. México: Interamericana.

Folkman, S. y Lazarus, R. (1986). An analysis of coping in a middleaged community sample. Journal of Health and Social Behavior. 21,219-239.

García, G. (1983). Ansiedad debilitadora y rendimiento escolar. Revista de Psicología de la PUCP. I(1),61-65.

Kerlinger, F. (1991). Investigación del comportamiento. México: Mc Graw-Hill.

Krantz, S. (1983). Cognitive appraisals and problem-directed coping: a prospective study of stress.

Journal of personality and social Psychology. 44, 638-643.

Lazarte, A. (1982). Unidimensionalidad versus multidimensionalidad de la escala de control interno-externo de Rotter. Tesis inédita de Bachillerato, Universidad Peruana Cayetano Heredia, Lima.

Lazarus, R. y Folkman, S. (1986). Estrés y Procesos Cognitivos. Barcelona: Martínez Roca.

Meza, A. (1987.). Relaciones entre asertividad y estilos cognitivos, afectivos y de orientación interpersonal. Tesis inédita de Maestría en Psicología, Universidad Peruana Cayetano Heredia, Lima.

Meza, A. (1988). Acerca de los estilos cognitivos, dependencia, independencia de campo. Revista de Psicología. V(5), 162-176.

Nawas, M. (1971). El Estilo de Vida 1. Introducción y estilo expresivo. Revista Latinoamericana de Psicología. 3(1), 91-107.

Quintana. A. (1998). Afrontamiento del estrés frente al examen en educación superior: Un estilo o un proceso? Tesis inédita de Maestría, UNMSM, Lima.

Rolter, B. (1966). Generalized expectances for internal versus external control of reinforcement. Psychological Monographs. 80, 1-28

Salazar, V. (1993). Relación entre estilos de afrontamiento al estrés y las dimensiones de personalidad neuróticas y extroversión; estudios realizados en estudiantes universitarios. Tesis inédita de Licenciatura, UPCH, Lima.

Spielberger, Ch. y Díaz-Guerrero, R. (1975). Inventario de ansiedad estado-rasgo.

México: El Manual Moderno.

Spielberger, Ch. (1980). Tensión y ansiedad. México: Harla. 